\title{
Koordination und Kontrolle
}

\section{Konflikte in der Zusammenarbeit von Haupt- und Ehrenamtlichen}

\author{
Uta Meier-Gräwe und Angelika Sennlaub
}

Prof. Dr. Uta Meier-Gräwe ist

Professorin für Wirtschaftslehre des Privathaushalts und Familienwissenschaft am Institut für Wirtschaftslehre des Haushalts und Verbrauchsforschung der Justus-Liebig-Universität Gießen. Internet http://wi.uni-giessen.de/wps/ fb09/home/meier

\section{Dr. Angelika Sennlaub ist}

Ökotrophologin und promovierte

Wohnökologin. Sie arbeitet freiberuflich mit dem Beratungsunternehmen "alltag von morgen « in Frankfurt am Main und bietet Forschung, Beratung und Fortbildungen zum Schwerpunkt Wohnen im Alter.

Internet http://www.alltagvonmorgen.de
Die Zusammenarbeit zwischen Ehrenamtlichen und Hauptamtlichen in der ambulanten Versorgung läuft nicht immer so gut, wie man es sich wünscht. Eine Studie in Gießen in Einrichtungen und Privathaushalten suchte nach den Ursachen und danach, wie man mit entsprechenden Konflikten umgehen kann.

Ehrenamtliche Leistungen werden gefordert, gefördert und breit (nicht nur) in Fachkreisen diskutiert. Angestrebt wird, nachlassende familiäre Sorgetätigkeiten abzufangen und die Versorgung besonders der älteren Hilfebedürftigen unserer Gesellschaft mit Blick auf die demografischen Veränderungen sicherzustellen.

Doch die Zusammenarbeit von hauptund ehrenamtlichen Mitarbeiterinnen und Mitarbeitern gestaltet sich oft problematisch. Was macht die Zusammenarbeit so schwierig? Wir wollen zunächst kurz Ursachen skizzieren und dann, daraus abgeleitet, aktuelle Lösungsstrategien vorstellen. Darüber hinaus weisen wir auf weitere Schritte hin, die aus unserer Sicht notwendig sein werden, um auf Ehrenamt als Stütze des Sorgesystems bauen zu können. (1)

Verwunderlich ist, dass trotz der breiten Diskussion bis heute nicht geklärt ist, was genau Ehrenamt ist: Manche zählen Leistungen gegen Entgelt (Übungsleiterpauschale o. Ä.) dazu, andere nicht; manche registrieren nur Leistungen an Fremden, andere beziehen nachbarschaftlichinformelle Tätigkeiten mit ein; wieder andere berücksichtigen den Einsatz im privathäuslichen Bereich, andere nicht. Eindrücklich kann das an zwei zentralen wissenschaftlichen Studien nachvollzogen werden; die neuere bezieht sich bewusst auf die ältere und fasst den Begriff ausdrücklich weiter. (2)

In dieser unklaren Begrifflichkeit liegt nach unserer Auffassung die Basis für die Schwierigkeiten, die zwischen haupt- und ehrenamtlich tätigen Personen bestehen: Solange nicht eindeutig vereinbart ist, um was es sich überhaupt handelt, werden es alle ausführenden Personen schwer haben, ihre Rolle im Alltag eingrenzen zu können.

Vor diesem Hintergrund steht der zentrale Konflikt: Es gibt eine deutliche Konkurrenz zwischen ehrenamtlich und hauptamtlich erbrachten Leistungen. Nach unserer Einschätzung sind es besonders fünf Faktoren, die die $\mathrm{Zu}$ sammenarbeit belasten:

1 Hauptamtlich Tätige befürchten, - dass ihre Arbeit durch Ehrenamt zumindest teilweise ersetzt wird. Diese Befürchtungen sind nicht unberechtigt, denn solche Fälle sind bekannt (3), und auch in den Interviews zur Studie gibt es mehrfach derartige Hinweise. Eine Seniorenberaterin etwa berichtet, sie informiere Hilfebedürftige je nach spezifischem Bedarf über die Möglichkeiten des Ehrenamts als Alternative zu professionellen Kräften. Ehrenamtliche Kräfte seien weitaus billiger, was für manche Leistungen durchaus relevant sei. "Wenn wir beraten, dass wir dann auch sagen: Sie haben 100 oder 200 Euro. Dann soll man schon gucken, ob man ne Fachkraft nimmt, die 22 oder 24 Euro die Stunde nimmt, oder ob man nen Ehrenamtlichen nimmt, der in drei Stunden 15 Euro kostet. Ja? «

7 Dazu kommt: Viele ehrenamtlich • engagierte Menschen möchten etwas Nützliches tun. Die Problematik, ob sie dabei originär bezahlte oder eher informell konnotierte Tätigkeiten übernehmen, ist vielen nicht präsent oder auch für einige nicht relevant. Der Geschäftsführer eines Wohlfahrtsverbandes berichtet beispielsweise von den Schwierigkeiten, die er besonders zu Beginn eines Einsatzes Ehrenamtlicher beobachtet: "Da kommen hoch engagierte Menschen, die sagen: So, ich bin jetzt da, ich mach hier alles. Ja? Also, dass dann der Hauptamtliche erst mal ausbremsen muss, gucken muss, wo ist eine Nische, wo passt du rein. " Eine Ehrenamtliche bestätigt im Interview die- 


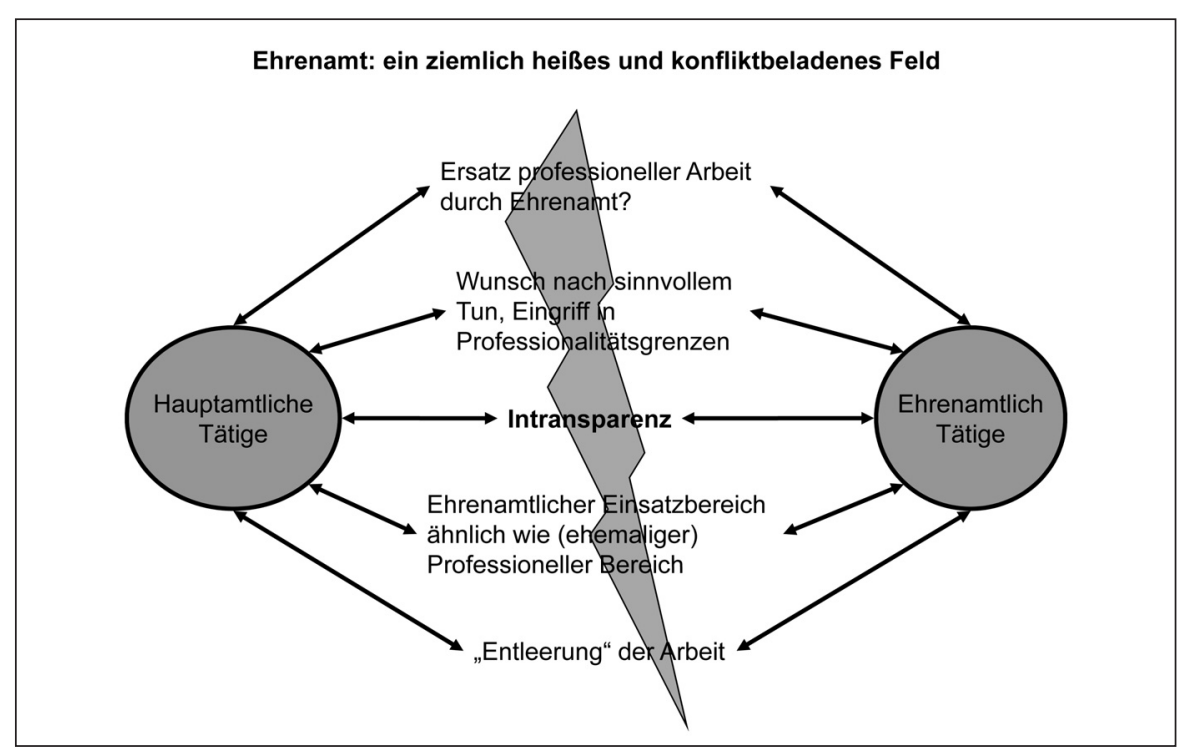

se Beobachtung: Sie sei bereit, alles das zu tun, was notwendig sei, solange sie es bewältigen könne. Auf Nachfragen bekräftigt sie: Ja, dazu zählten auch hauswirtschaftliche oder pflegerische Tätigkeiten, von denen sie genug wisse.

3 Verstärkend kommt hinzu, dass vie- le freiwillig Tätige mit ihrem Ehrenamt an vorherige berufliche Arbeitsbereiche anknüpfen. Damit sind sie mit der Kompetenz einer Fachkraft unbezahlt tätig, gleichzeitig aber anderen Fachkräften unterstellt, die sie zudem zumindest teilweise für weniger kompetent oder erfahren als sich selbst halten. Ein Wissenschaftler, der sich umfänglich mit Engagementformen beschäftigt hat, berichtet entsprechend: »Das sind ja oft die Profis, die Geld kriegen und in ihrer Freizeit dann noch das (Ehrenamt) machen. (...) Das ist ein sehr häufiges Phänomen. « $\triangle$ Viertens befürchten professionelle • Kräfte eine »Entleerung « ihrer Tätigkeit. So berichtet der Wissenschaftler, der selbst ehrenamtlich in der Hospizarbeit tätig ist, von solchen Schwierigkeiten: »In der ganzen Hospizarbeit gibt es die Äußerungen: Ja, wir Pflegekräfte dürfen die Windeln wechseln, und dann kommt der Hospizhelfer und führt die schönen Gespräche, und die nehmen uns das weg, was wir eigentlich machen wollen.«

5 Was die Situation sicherlich er• schwert ist die Beobachtung, dass über den Konflikt nicht offen gesprochen wird. "Das ist ein ziemlich heißes und konfliktbeladenes Feld «, sagt etwa einer der Geschäftsführer der Wohlfahrtsverbände, konkreter will er aber nicht werden. Obwohl mehrere ansprechen, dass es Probleme gibt, wird bei Nachfragen überwiegend ausgewichen.
Strategie: enge Begleitung und Kontrolle durch Ehrenamtskoordinatorinnen oder -koordinatoren

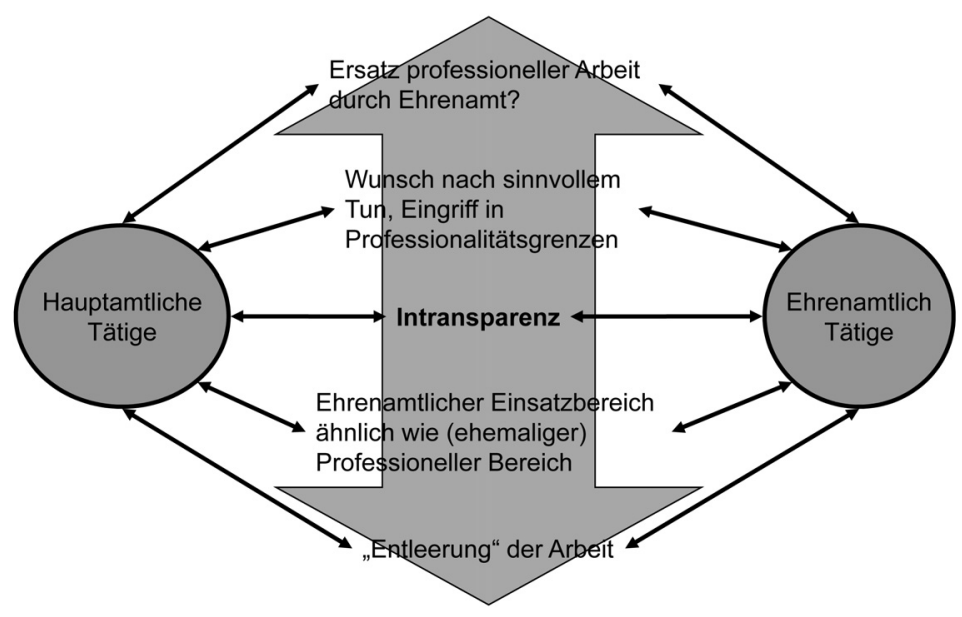

\section{Strategie: enge Begleitung und Kontrolle}

Lösungen zur guten Zusammenarbeit werden vor allem von denjenigen entwickelt, die die Einsätze koordinieren: Sie fühlen sich für beide Personengruppen gleichermaßen verantwortlich und bemühen sich, potenzielle Probleme so weit wie möglich zu vermeiden. Die wesentliche Strategie der Ehrenamtskoordinatorinnen und Ehrenamtskoordinatoren liegt darin, einen engen Kontakt zu halten und den Einsatz regelmäßig zu kontrollieren.

Beim Einsatz in einer Institution muss sowohl zu der ehrenamtlich tätigen Person wie auch zur Verantwortlichen vor Ort ein enger Kontakt gehalten werden. In einem Interview wird beispielsweise eine schwierige Situation und die Lösungsstrategie geschildert: Eine Ehrenamtliche sollte in der Bibliothek einer Schule eingesetzt werden, weil es ihr Wunsch war, im Rahmen ihres Ehrenamtes mehr mit Kindern in Kontakt zu kommen. Dennoch war sie mehr und mehr in der Küche beschäftigt und wurde zum Servieren des Essens eingesetzt. Die Ehrenamtskoordinatorin, die sie in diese Schule vermittelt hatte, suchte daraufhin das Gespräch mit der Ehrenamtlichen: »Ich sagte dann, wir müssen schon Ihre Zuständigkeiten ganz klar festlegen. Sie sagte dann: Ich kann auch einkaufen, ich kann auch dies noch machen und die Lebensmittel und - sie kommt auch noch aus dem Bereich und sagt: Da kann man wesentlich gesünder und kostengünstiger noch einkaufen. Was natürlich auch ein ganz - kritisches Ding ist, wo man sie auch ein bisschen in ihren Bereich reinsortieren muss. Also, sie meint das total gut, aber -. Also, von daher braucht Ehrenamt eine sehr gute Begleitung, und dann muss auch geklärt werden, wer ist auch dafür (Kontrolle des Einsatzes) zuständig. "Die Koordinatorin suchte zusätzlich den Kontakt zur Leitung der Schule, um gemeinsam mit ihr die Problematik zu klären, dass die Ehrenamtliche zunehmend wie eine Fachkraft ohne Lohn arbeite. Die beiden verabredeten eine enge Begleitung der Situation.

Ein Einsatz in der privaten Häuslichkeit ist von vornherein weit weniger kontrollierbar als ein Einsatz, bei dem mehrere Menschen anwesend sind oder der direkt in einen Arbeitsprozess eingebunden ist. Die Strategie muss deshalb darauf zielen, 
auch hier eine größtmögliche Transparenz zu schaffen. Auch hier wird ein enger direkter Kontakt gehalten, der nicht nur Kontrollfunktion, sondern auch eine unterstützende Aufgabe hat: Sowohl die ehrenamtlich tätige Person als auch die älteren Hilfebedürftigen müssen begleitet werden, damit diese "geschlossene « Situation im Privatbereich offener gestaltet und Missverständnisse hinsichtlich des Aufgabenbereichs behoben werden können.

In einem Wohlfahrtsverband wird deshalb jeder Einsatz vor allem zu Beginn eng begleitet: »Wir haben ne Stabsstelle hier im Verband, bei der auch Ehrenamt angesiedelt ist. (...) Die Mitarbeiterin geht immer hin, bevor sie einen Einsatz vermittelt, guckt sich das an, wie das bei denen zu Hause ist, was wollen die Leute, was gibt es zu berücksichtigen, dann führt sie Gespräche mit den Ehrenamtlichen, dann werden die geschult, und dann wird versucht, das optimal zueinander zu bringen. «

Auch mehrere Vertreterinnen von Beratungs- und Koordinierungsstellen für ältere und pflegebedürftige Menschen in der Stadt und im Landkreis Gießen betonen die Notwendigkeit, vor allem zu Beginn Einsätze im privathäuslichen Bereich eng zu begleiten und regelmäßig Rücksprache sowohl mit den ehrenamtlich Tätigen wie auch mit den privaten Auftraggeberinnen und Auftraggebern zu halten. Sie haben einen klaren Ablauf erarbeitet, wer wann wie oft mit wem spricht, um von Anfang an Probleme offen anzusprechen und gemeinsam nach Lösungen $\mathrm{zu}$ suchen.

\section{Strategien: Qualifizierung, Vereinbarung und Transparenz}

Was fehlt, sind vor allem drei Ansätze:

- Erstens muss die Qualifizierung von ehrenamtlichen Kräften ein Modul zur Problematik der Zusammenarbeit enthalten. Dadurch werden Ehrenamtliche sensibilisiert, schwierige Situationen zu erkennen und angemessen damit umgehen zu können.

- Zweitens wird es hilfreich sein, »ehrenamtliche Inhalte« zu vereinbaren. Wenn durch das Ehrenamt reguläre Arbeitsplätze zumindest teilweise ersetzt werden, wird es schwierig sein, eine gute Zusammenarbeit von Haupt- und Ehrenamt zu erreichen. Falls das gewollt ist, muss es zumindest offen for-

\section{Strategien, die ausgebaut werden müssen: Qualifizierung und Transparenz}

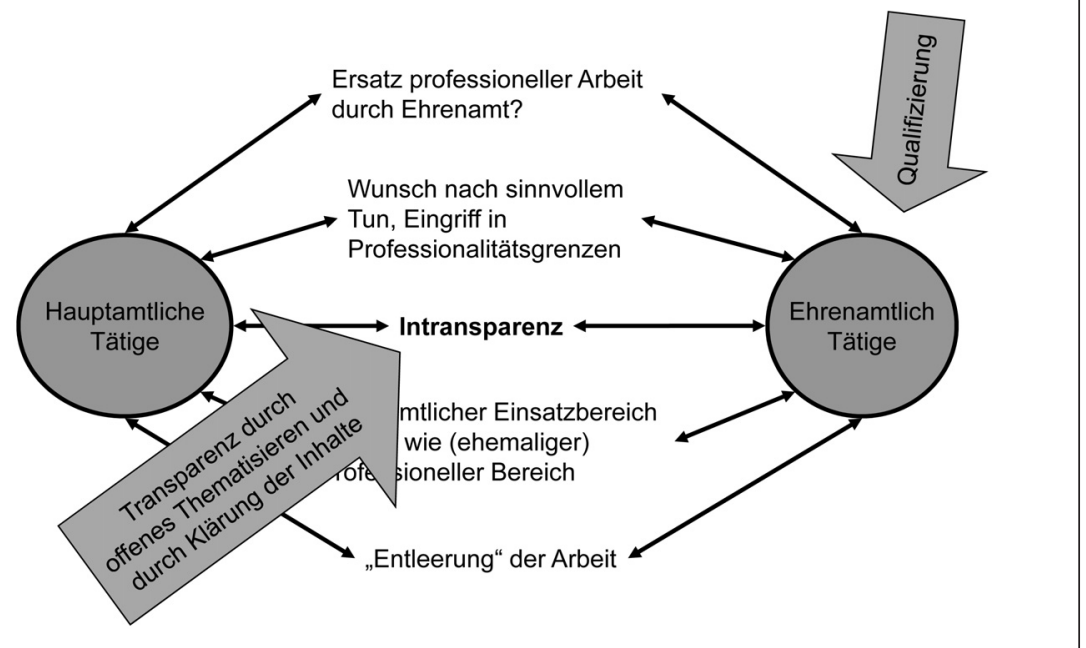

muliert werden, damit die Beteiligten die Chance bekommen, offen darüber diskutieren zu können.

- Drittens muss die Problematik insgesamt offener thematisiert werden. Nur so können Befürchtungen von tatsächlichen Schwierigkeiten unterschieden und die Diskussion versachlicht werden. Das wird wesentlich sein, um das teilweise unbekannte konfliktreiche Feld zu entschärfen.

\section{Anmerkungen}

(1) Der Text gibt einen Teilbereich der Studie »Wirkungsanalyse und Perspektiven der BeKo-Stelle für ältere und pflegebedürftige Menschen in der Stadt und im Landkreis Gießen unter besonderer Berücksichtigung der Einbeziehung von bürgerschaftlichem Engagement (WiPeB-Studie) « wieder, die von der Justus-Liebig-Universität Gießen am Lehrstuhl für Wirtschaftslehre des Privathaushalts und Familienwissenschaft, Prof. Dr. Uta Meier-Gräwe, im Auftrag des Hessischen Ministeriums für Arbeit, Familie und Gesundheit, Abteilung II 5 Seniorinnen und Senioren, Dr. Marie-Luise Marx, von September 2008 bis Juli 2010 durchgeführt und von Dr. Angelika Sennlaub bearbeitet wurde. Die Fragestellung wurde qualitativ bearbeitet, das Ergebnis im August 2010 dem Ministerium vorgelegt. Im Rahmen der Studie wurden 29 Interviews mit 42 Personen ausgewertet. Interviewpartnerinnen und Interviewpartner waren haupt- und ehrenamtliche Akteurin- nen und Akteure der Altenhilfe unterschiedlicher Profession und Funktion, Angehörige der Wissenschaft und ältere hilfebedürftige Personen beziehungsweise ihre Angehörigen.

(2) Enquete-Kommission 2002, BMFSFJ 2009.

(3) Z. B. Panorama 2010.

\section{Literatur}

BMFSFJ Bundesministerium für Familie, Senioren, Frauen und Jugend (Hg.) (2009): Bericht zur Lage und zu den Perspektiven des bürgerschaftlichen Engagements in Deutschland. Bearbeitet vom Wissenschaftszentrum Berlin für Sozialforschung (WZB), Projektgruppe Zivilengagement, Mareike Alscher, Dietmar Dathe, Eckhard Priller (Projektleitung), Rudolf Speth. Berlin.

Enquete-Kommission "Zukunft des Bürgerschaftlichen Engagements ", Deutscher Bundestag (Hg.) (2002): Bürgerschaftliches Engagement: Auf dem Weg in eine zukunftsfähige Bürgergesellschaft. Leske + Budrich, Opladen.

Panorama 2010: Beitrag in der Sendung Panorama am 1. Juli 2010 mit dem Titel »Ehrenämter bei Caritas \& Co: Tarnung von Billigjobs «, abrufbar unter http://daserste.ndr.de/panora$\mathrm{ma} / \mathrm{media} /$ panorama494.html.

Die WiPeB-Studie ist abrufbar auf den Internetseiten der Autorinnen: http://wi.uni-giessen.de/wps/fb09/ home/meier und http://www.alltagvonmorgen.de. 